\title{
Efficacy and safety of Naoxintong Capsule for treating Chronic Stable Angina: study protocol for a randomized controlled trial
}

\section{Gao Huanjia}

Guangzhou University of Chinese Medicine

\section{Cai Hairong}

Guangzhou University of Chinese Medicine

\section{Zhuang Jieqin}

Guangdong Hospital of Traditional Chinese Medicine

\section{Dai Xingzhen}

Guangdong Hospital of Traditional Chinese Medicine

\section{Fu Xue}

Guangdong Hospital of Traditional Chinese Medicine

\section{Zhang Weizhang}

Guangdong Hospital of Traditional Chinese Medicine

\section{Bojun Chen ( $D 1198039070 @ q q . c o m$ )}

Guangdong Hospital of Traditional Chinese Medicine

\section{Study protocol}

Keywords: Chronic stable angina, Naoxintong Capsule, traditional Chinese medicine, Randomized controlled trial

Posted Date: March 8th, 2021

DOI: https://doi.org/10.21203/rs.3.rs-58168/v1

License: (c) (i) This work is licensed under a Creative Commons Attribution 4.0 International License. Read Full License 


\section{Abstract}

Background Cardiovascular disease is the leading cause of mortality and morbidity worldwide, Chronic stable angina (CSA) is the main symptom of myocardial ischemia, causes increased risk of major cardiovascular events such as sudden cardiac death and myocardial infarction.Naoxintong (NXT)Capsule is a classical traditional Chinese medication used to treat CSA, however, few evidence to support the wide utility of NXT capsule for the treatment of CSA. We design this study to evaluating the efficacy and safety of NXT capsule versus placebo in patients with CSA. Methods/design This is a multicenter, randomized, double-blind, placebo-controlled clinical trial. A total of 260 eligible participants will be enrolled. The participants will be randomized assigned in an equal ratio to groups receiving either NXT or placebo for 12 weeks. After a 2-week run-in period, they will receive either NXT or placebo (3 pills, 3 times daily) for 12 weeks. The primary outcome is therapeutic efficacy. Secondary outcome measures include the quantitative score of TCM syndromes, severity grading of angina pectoris, the number of angina pectoris per week, nitroglycerin dosage, score of seattle angina scale, serum homocysteine, incidence of cardiovascular events. Safety outcomes and adverse events will be monitored throughout the trial. Discussion We designed this study in accordance with principles and regulations issued by the China Food and Drug Administration (CFDA). The results will provide clinical evidence of the efficacy and safety of NXT Capsule in the treatment of CSA.

Trial registration: ChiCTR2000034871.

\section{Background}

Cardiovascular disease, despite many advances in its diagnosis and treatment, remains the leading cause of morbidity and mortality worldwide, leading to over 17.3 million deaths per year and perhaps growing to over 23.6 million deaths per year by 2030.[1,2] CSA is a major symptomatic presentation in about $50 \%$ patients with coronary heart disease (CHD)[3], which is characterized by chest pain or discomfort caused by a temporary disruption in the flow of blood and oxygen to the heart. CSA always triggered by exertion , rest or sublingual nitroglycerin usually relieves angina within 30 seconds to several seconds. $[4,5]$ The goals of treating CSA are to improve quality of life and minimize the risk of cardiovascular events [6].At present, Guideline-directed therapy for CSA includes nitrates, beta-blockers, calcium channel blockers, angiotensin-converting enzyme inhibitors statins, and antiplatelet agents. [4]. In addition, lifestyle changes, such as maintaining a healthy weight, consuming a low-fat diet, discontinuing the use of tobacco products, and finding ways to reduce stress are also encouraged.[7]

Chinese patent medicines (CPMs) are widely used in China as adjuvant therapies for western medicines. TCM has the advantage of its multi-target and multi-link therapeutic effects and less adverse reactions than western therapies, and aslo has a long history in treating CSA, NXT is a commercial medicinal product approved by the China Food and Drug Administration which is widely used in the treatment of stroke and coronary heart disease, existing studies have demonstrated NXT can help control angina pain, NXT combined with anti-anginal medications have been proved effective and safe in the treatment of CSA. $[8,9]$. NXT is made up of several traditional Chinese medicines which can activate blood circulation and 
remove blood stasis.(Table 1).Although there are some Cochrane reviews have shown the potential benefit of TCM in treating CHD, the current research is methodologically weak, it is meaningful to accumulate more evidence from high-quality trials to demonstrate the clinical use of Chinese patent medicine. We designed a randomized controlled trial for the purpose of providing high-level evidence for the clinical application of NXT capsule and clarifying the efficacy and safety of NXT capsule in the treatment of patients with CSA through multicenter, randomized, double-blind, placebo-controlled, clinical trial.

Table 1 Pharmacological effects of each ingredient in NXT

Ingredient

Pharmacological

effects

Red peony root, Salvia miltiorrhiza, Angelica sinensis, Chuanxiong,

Peach kernel, Safflower, Caulis spatholepis, Earthworm, Leech

Astragalus, Oxknee

Invigorate the circulation of blood

Cassia twig, Mulberry twig

Boost immunity

Dredging collaterals

\section{Methods/design}

This study is multicenter, randomized, double-blind, placebo-controlled, clinical trial. A total of 260 eligible participants will be enrolled. The participants will be randomized assigned in an equal ratio to groups receiving either NXT or placebo for 12 weeks. After a 2-week run-in period, they will receive either NXT or placebo ( 3 pills, 3 times daily) for 12 weeks. Efficacy and safety data will be obtained during the treatment. A flow chart of the trial is illustrated in Figure 1.

\section{Ethics}

This trial has been registered in the China Clinical Trial Registry (ChiCTR2000034871). This trial is reported in accordance with the Standard Protocol Items: Recommendations for Intervention Trials (SPIRIT) guidelines[10,11].Ethical approval has been obtained from the Ethics Committee of the Guangdong Provincial Hospital of Traditional Chinese Medicine (BF2020-152-01). Participants who meet all of the inclusion criteria will be asked to sign an informed consent before the trial that will contain the details about the trial such as inclusion criteria, exclusion criteria, therapeutic interventions, scheduling, trial benefits, and possible risks of this trial. Participants have the right to desert throughout the trial.

\section{Participants and recruitment}

Participants will be recruited through Internet advertisement and posters in the community and selected hospitals. A total of 240 eligible participants will be recruited in the following six hospitals: (1) Guangdong Provincial Hospital of Traditional Chinese Medicine, (2) The First Affiliated Hospital of Sun Yat-sen University, (3) Zhujiang Hospital, (4) Southern Medical University Hospital, (5) Shenzhen Longgang District 
Hospital of Traditional Chinese Medicine and (6) Yangjiang People's Hospital. The first center will recruit 80 patients, with 32 for each of the remaining centers.

\section{Eligibility criteria}

Eligible participants are those who fulfill all of the listed inclusion criteria and do not have any of the exclusion criteria.

\section{Diagnostic criteria}

The diagnostic criteria for CHD

(1)A history of myocardial infarction, with or without revascularization (percutaneous coronary intervention (PCI) or coronary artery bypass grafting) treatment;

(2) Coronary angiography confirmation or computed tomography coronary angiography confirmation of stenosis greater than $50 \%$ of at least 1 major branch of the coronary artery luminal diameter, with or without revascularization;

(3) Noninvasive imaging stress test diagnostic of CHD.

A patient meeting at least one of the above criteria is considered to be diagnosed with CHD.

The diagnostic criteria for CSA

Diagnostic criteria for CSA were determined according to the Chinese Medical Association's 2007 Guidelines for the Diagnosis and Treatment of Chronic Stable Angina[12], 2013 ESC guidelines on the management of stable coronary artery disease[13],The classification of angina referred to the Canadian Cardiovascular Society (CSS) Functional Classification of Angina.

\section{Inclusion criteria}

(1)A diagnosis of CHD and the provision of exact imaging information (coronary angiography or computed tomography coronary angiography confirmation of stenosis greater than $50 \%$ of at least one major branch of the coronary artery luminal diameter, or nuclear perfusion scan diagnosed as CAD). CCS classification of angina grade II

(2)The onset of angina pectoris $\geq 3$ months previous and the frequency of angina attack $\geq$ twice a week

(3)Age between 35 and 75, regardless of gender

(4)Signed informed consent by participants or surrogates

\section{Exclusion criteria}


(1)Uncontrolled or mismanaged blood pressure and blood glucose. Severe cardiopulmonary insufficiency, or severe arrhythmia (rapid atrial fibrillation and flutter, paroxysmal ventricular tachycardia second degree and greater than a second degree atrioventricular (AV) block). Acute $\mathrm{MI}$ in the past 2 months, or has undergone coronary revascularization in the past 12 months.

(2)Renal dysfunction, male serum creatinine $>2.5 \mathrm{mg} / \mathrm{dl}(>220 \mu \mathrm{mol} / \mathrm{L})$ or female serum creatinine $>$ $2.0 \mathrm{mg} / \mathrm{dl}(>175 \mu \mathrm{mol} / \mathrm{L})$. Serious liver disease邓expression of aminotransferase (ALT) and aspartate aminotransferase (AST) of 1.5 times higher than the normal upper limit $\rrbracket$.

(3)Factors that precluded satisfactory interpretation of the electrocardiogram (ECG) (e.g. digoxin therapy, left bundle branch block, implanted with pacemaker, left ventricular hypertrophy, or electrolyte disturbance).

(4)Complications with a serious bone joint disease or other comorbidities that may interfere with ability to perform required ETT.

(5)Patients planning to undergo coronary revascularization during the study period.

(6)Patients who might be allergic or are known to be allergic to ingredients of the study drug.

(7)pregnant, pregnancy planners or lactating women.

(8)Patients who are allergic to NXT capsule.

(9)Substance abuse, alcohol and drug dependence in the last 2 years.

(10)Patients who participated in other clinical drug trials within 1 month.

\section{Withdrawal criteria}

The rejection criteria include the following:

(1)Patients who experience serious complications or rapid deterioration of the condition throughout the trial.

(2)Serious adverse events (AEs) occurring, which would result in treatment being stopped according to the doctors' decision.

(3)Participants with vital deviations in the implementation of the study, such as poor compliance and difficulty in evaluating drug effects

(4)Patients who quit this clinical trial voluntarily

\section{Sample size}


The sample size was estimated to treat the symptoms of angina pectoris for 12 weeks as the main effect index according to the statistical requirements. According to the literature, the total effective rate of basic treatment + placebo was $67.5 \%$, and the total effective rate of basic treatment + test drug was estimated to be 84.3 . $\%$, let $a=0.05,1-\beta=0.2$, according to the test group: control group $=1: 1$ set, after calculation, the minimum sample size that meets the statistical requirements is 98.4 cases per group, considering no more than $20 \%$ the shedding rate was 120 cases in each group, a total of 240 cases.

\section{Randomization and blinding}

A total of 240 participants will be randomized in a 1:1 ratio assigned to the NXT group and the control group by the method of using 240 opaque envelopes. Half of them are labeled with NXT group, and half of them are control group. After all the envelopes have been sealed, they will be mixed evenly and distributed to each research center. When a sub-center accepts an eligible patient, the baseline information such as the subject's hospital ID number, name, age and gender will be recorded. All participants, investigators, and attending physicians will be blinded to the treatment assignments until the research is finished. Only drug administrators and dispensing nurses can open the envelope to check the group allocation and perform the interventions according to the instructions of this study, All investigators, outcome assessors and data analysts will be blinded to collect and summarize which is only based on a subject's baseline information until the completion of the visit and analysis.

\section{Interventions}

Eligible patients will be randomly allocated to a treatment group or a control group in an equal ratio. In order to ensure the safety of participants, enrolled patients in both control group and treatment group will receive standard conventional therapies, under the Chinese Guidelines for the Diagnosis and Treatment of CSA (2007) [14]. Routine medications in the trial as follows:

(1)antiplatelet agents: aspirin (75-100 mg, once per day) or clopidogrel;

(2)Lipid-lowering agents (statins): atorvastatin (10-20 mg, once per day) or simvastatin (20-40 mg, once per day).

(3)Anti-angina agents: $\beta$-blockers (metoprolol $50-200 \mathrm{mg}$, once per day, or analogous agents); long-acting nitrates (isosorbide mononitrate 40-60 mg, once per day); or calcium channel blockers (amlodipine 5$20 \mathrm{mg}$, once per day).

On the basis of conventional western medicine treatment, the experimental group will receive NXT capsule for 12 weeks, 3 pills/day, taken orally, after meals; while the control group will receive placebo capsule, the placebo capsule simulator is similar to the NXT capsule, with a comparable appearance, the primary content of the placebo capsule is starch, the control group will receive NXT capsule simulator for 12 weeks, 3 pills/day, taken orally, after meals,

\section{Efficacy assessment}


Primary outcome: The primary outcomes of this study include changes of curative effect of angina pectoris symptoms: The number of angina attacks, degree of pain, duration, as well as the dosage of nitroglycerin are used as indicators for scoring. Score ranges from 0 to 15, with higher score indicate more severe angina. (Table 2)

Table 2 Measurement items and points of data capture.

\begin{tabular}{|c|c|c|c|c|c|}
\hline Study phase & & in period & intervention pe & & \\
\hline Time & $\frac{\text { Visit 1 }}{-14 \pm 2 \text { davs }}$ & $\begin{array}{c}\text { Visit 2 } \\
-4 \square 0 \text { davs }\end{array}$ & $\frac{\text { Visit3 }}{4 \text { 4weeks+4davs }}$ & $\begin{array}{c}\text { Visit4 } \\
\text { 8weeks +4davs }\end{array}$ & $\begin{array}{c}\text { Visit5 } \\
\text { 12weeks+4davs }\end{array}$ \\
\hline Baseline data collection & & & & & \\
\hline Informed consent & & & & & \\
\hline Demographic data & & & & & \\
\hline Concomitant disease and & & & & & \\
\hline Inclusion/exclusion criteria & & & & & \\
\hline Screen index & & & & & \\
\hline $\begin{array}{l}\text { Urinary pregnancy test } \\
\text { Safety evaluation }\end{array}$ & & & & & \\
\hline Vital signs & & & & & \\
\hline $\begin{array}{l}\text { Blood and urine routine, } \\
\text { stool routine+occult } \\
\text { blood, fasting blood } \\
\text { glucose }\end{array}$ & & & & & \\
\hline $\begin{array}{l}\text { Liver funtion₫ALT,AST,TBIL } \square \\
\text { and renal function test } \\
\square \mathrm{BUN} \square \mathrm{Cr} \square\end{array}$ & & & & & \\
\hline $\begin{array}{l}\text { Coagulation function test } \\
\text { Blood lipid level }\end{array}$ & & & & & \\
\hline ECG & & & & & \\
\hline Efficiency evaluation & & & & & \\
\hline TCM syndrome score & & & & & \\
\hline $\begin{array}{l}\text { Scores of angina symptoms } \\
\text { CCS anqina classification }\end{array}$ & & & & & \\
\hline $\begin{array}{l}\text { CCS angina classification } \\
\text { Number of episodes of AP }\end{array}$ & & & & & \\
\hline $\begin{array}{l}\text { Number of episodes of AP } \\
\text { per week } \\
\text { Nitroglycerin consumption }\end{array}$ & & & & & \\
\hline $\begin{array}{l}\text { Nitroglycerin consumption } \\
\text { Score of seattle angina scale }\end{array}$ & & & & & \\
\hline Homocysteine & & & & & \\
\hline Other work & & & & & \\
\hline Random grouping & & & & & \\
\hline Dispense drug & & & & & \\
\hline Recovery drug & & & & & \\
\hline $\begin{array}{l}\text { Aes records } \\
\text { Fvaluate the clinical efficas }\end{array}$ & & & & & \\
\hline $\begin{array}{l}\text { Evaluate the clinical efficacy } \\
\text { and adherence }\end{array}$ & & & & & \\
\hline
\end{tabular}

\section{Secondary outcomes}

(1)Therapeutic effect of TCM syndromes: The TCM syndromes scoring system used in this study will follow the guidelines of clinical research on the treatment of coronary heart disease (chest pain) with new Chinese medicine, in which all symptom and sign scores are graded (Table 3). The symptoms of CSA include: chest pain, chest tightness, breathlessness, palpitations, mental fatigue, aversion to cold, and cold limbs, lumbar and knee soreness, spontaneous sweating, and insomnia. The score ranges from 0 to 27.0 
indicate asymptomatic, 1 for mild, 2 for moderate and 3 points for severe. The higher the score, the severe it is.

Table 3 Symptom and sign scores

\begin{tabular}{|c|c|c|c|c|}
\hline \multirow[t]{2}{*}{ Symptom or sign } & \multicolumn{4}{|l|}{ score } \\
\hline & Asymptomatic(0) & Mild(1) & Moderate(2) & Severe $13 \square$ \\
\hline Chest pain(angina) & none & $\begin{array}{l}\text { Occasionally, } \\
\text { self-medication }\end{array}$ & $\begin{array}{l}\text { Frequently } \\
\text { obvious when } \\
\text { moving }\end{array}$ & $\begin{array}{l}\text { Sustained, } \\
\text { can't insist on work }\end{array}$ \\
\hline Breathless & 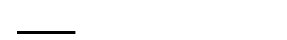 & ـ & 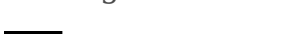 & 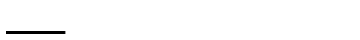 \\
\hline Palpitations & 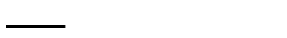 & 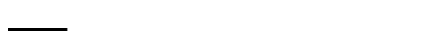 & - & 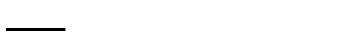 \\
\hline Chest tightness & - & 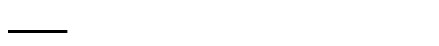 & 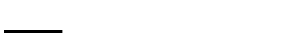 & - \\
\hline Mental fatigue & & 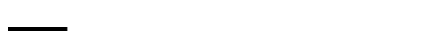 & 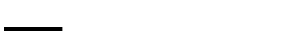 & $\square$ \\
\hline Aversion to cold and & - & - & Frequently & Severe \\
\hline cold limbs & & & $\begin{array}{l}\text { Need to add } \\
\text { clothes }\end{array}$ & $\begin{array}{l}\text { Can not ease when } \\
\text { adding clothes }\end{array}$ \\
\hline $\begin{array}{l}\text { Lumber and knee } \\
\text { soreness }\end{array}$ & 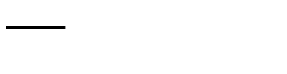 & 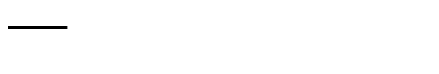 & - & $\longrightarrow$ \\
\hline Spontaneous sweating & ـ & Occasionally, & Frequently & Severe, \\
\hline & & Aggravation when moving & $\begin{array}{l}\text { When a little } \\
\text { activity }\end{array}$ & When no movement \\
\hline insomnia & - & Mild & Moderate & Severe, \\
\hline & & $\begin{array}{l}\text { Difficult to sleep, but } \\
\text { hardly affected }\end{array}$ & $\begin{array}{l}\text { Cannot sleep for } \\
\text { hours }\end{array}$ & cannot sleep all night \\
\hline
\end{tabular}

(2)Grading changes of severity of angina pectoris: According to CCS Angina Severity Classification Standard. I: General physical activity does not cause angina pectoris, angina with strenuous, rapid, or prolonged exertion. II: Slight limitation of ordinary activity, angina when walking upstairs briskly, or walking on a cold or windy day. III: marked limitation, angina when walking at anormal pace up flight of stairs, or walking 1-2 blocks distance. IV: Angina on minimal exertion or even at rest.

(3) Frequency of angina pectoris episodes per week.

(4) The dosage of Nitroglycerin;

(5) Seattle Angina Questionnaire (SAQ): The SAQ quantifies patients' physical limitations caused by angina, the frequency of and recent changes in their symptoms, their satisfaction with treatment, and the degree to which they perceive their disease to affect their quality of life. Each scale is transformed to a score of 0 to 100, where higher scores indicate better function (eg, less physical limitation, less angina, and better quality of life).

(6) Incidence of cardiovascular event during the 12 weeks such as developing into unstable angina, acute myocardial infarction and even death.

\section{Safety outcomes}

Safety outcomes includes vital signs (temperature, heart rate, breathing, and blood pressure after 10 min of rest), coagulation function test, liver and renal function, routine blood tests, urine and stool tests, and electrocardiograph (ECG)

\section{Management and statistical analysis}


Statistical analysis will be performed by the Drug Clinical Research Center of Guangzhou University of TCM, 2 independent trained data administrators will read the CRFs and record the data on the EpiData 3.1 software, the study monitor will cross-check the electronic case report forms against the data administrators' records on the purpose of ensuring the accuracy and reliability of the data. The database will be locked after blinding state data review and the statistical analysis can be performed only with the main investigator, sponsor, and statistical analyst.

Statistical analysis will be performed by a statistician blinded to the whole trial process using SAS 9.3 statistical software packages. The full analysis set (FAS) is the primary analysis set, in which one group receive NXT capsule treatment and the other placebo treatment. For the evaluation of curative effect in this trial, the per-protocol set (PPS) was used. Efficacy assessment will be performed through FAS and PPS. Safety evaluation will also be conducted. Continuous variables will be described using means and standard deviations and tested with Student's $t$ tests. Categorical variables are expressed in frequency counts and percentages and tested with chi-squared tests. More details will be described in a formal statistical analysis plan. Baseline balance between groups will be performed by a chi-square test or analysis of covariance (ANOVA). All collected data will be processed by professional statisticians using SAS 9.3 software; a two-sided $P$ value of $<0.05$ is considered statistically significant.

\section{Discussion}

This study is a randomized, double-blind, placebo-controlled trial designed for NXT capsule to evaluate the efficacy and safety in the treatment of CSA. Coronary heart disease is the leading cause of death worldwide, the financial impact of treating CHD and angina can be large[15, 16].According to World Health Organization statistics, CHD accounts for 17.3 million deaths/year, the annual global CVD mortality could be rising to 23.6 million by 2030[17].CSA affects a large population across the world, and thus has a high mortality rate[18, 19].This study focus on the improvement of participants' symptoms, it is meaningful in the evaluation of the efficacy on CSA.[20] TCM has a considerable role in alleviating symptoms and maximizing quality of life of CSA patients. Hence, the efficacy of angina pectoris symptoms and TCM syndromes will be selected as therapeutic indicators, since they are correlated to patients' symptoms and quality of life.

NXT Capsule, a modern patent traditional Chinese medicine, is mainly made up of Huangqi, Danshen, Quanxie, Shuizhi and other sixteen herbs. It is extensively used to treat coronary heart disease, stroke and other cardiovascular and cerebrovascular diseases, with the activity of anti-coagulation, anti-inflammatory, protecting endothelial cells, anti-atherogenic and plaque stabilization. Previous studies have shown that NXT capsule is safe and effective in cardiovascular protection. NXT capsule is known as a potent adjuvant treatment for CSA[21-23]. On the purpose of determining the efficacy and safety of NXT capsule combined with conventional therapy for patients with CSA , we designed this multicenter, double-blind, placebo-controlled randomized clinical trial in accordance with the Consolidated Standards of Reporting Trials (CONSORT) guidelines and the "One study, one primary outcome" clinical trial methodology [24].We aim to provide new and high-quality evidence in NXT capsule treatment for patients with CSA. 
Furthermore, this protocol presents a detailed and practical methodology for future clinical trials of developing TCM.

\section{Trial Status}

The version of the protocol is V1.0 and the date is May 3, 2020, and the recruitment date is from2020/07/21 To 2022/07/21. Currently, patient recruitment for the trial is on-going.

\section{Abbreviations}

AEs: adverse events;CSA: Chronic stable angina;CHD: coronary heart disease;CONSORT: Consolidated Standards of Reporting Trials;CSS: Canadian Cardiovascular Society;CPMs: Chinese patent medicines CFDA: China Food and Drug Administration;ECG: electrocardiograph;FAS: full analysis set ;PPS: perprotocol set;PCI: percutaneous coronary intervention;SAQ: Seattle Angina Questionnaire;TCM: traditional Chinese medicine;NXT: naoxintong.

\section{Declarations}

\section{Acknowledgements}

All costs associated with the study are funded by the China Shaanxi Buchang Pharmaceutical Co. LTD (sponsor), who did not participate in any steps of the study design, data collection and statistical analysis of the trial. We are very grateful to those project leaders from the other five centers. We also express full thanks to the Contract Research Organization (CRO) and all other participants in this study.

\section{Funding}

The trial was sponsored by the China Shaanxi Buchang Pharmaceutical Co. LTD The authors also acknowledge the support of the other two funds: the National Natural Science Foundation of China (81273961 and 81303117 ). The funders had no role in the design of the study,analysis, collection, and interpretation of the data, or the writing and decision for publication of the manuscript. The funder does not have ultimate authority over any of these activities.

\section{Availability of data and materials}

All data will be made available.

\section{Authors' contributions}

$\mathrm{GH}, \mathrm{CH}$, and $\mathrm{ZJ}$ contributed to the design and development of the trial. $\mathrm{CB}$ is the principal investigator of this study. GH drafted the initial manuscript, and ZW revised it carefully. DX and FX participated in the design and coordination of the trial as well as in recruiting patients. All authors reviewed the content and approved the final version. 


\section{Competing interests}

The authors declare that they have no competing interests.

\section{Consent for publication}

Not applicable.

\section{Ethics approval and consent to participate}

The protocol, informed consent form, and recruitment poster were reviewed and approved by the Ethics Committee of the Guangdong Provincial Hospital of Traditional Chinese Medicine (No. BF 2020-152-01), the Ethics Committee of the The First Affiliated Hospital of Sun Yat-sen University, the Ethics Committee of the Zhujiang Hospital, the Ethics Committee of the Southern Medical University Hospital, the Ethics Committee of the Shenzhen Longgang District Hospital of Traditional Chinese Medicine, the Ethics Committee of the Yangjiang People's Hospital.Written informed consent will be obtained from each participant prior to enrollment.

\section{References}

1. Zhao L, Li D, Zheng H, Chang X, Cui J, Wang R, Shi J, Fan H, Li Y, Sun X et al: Acupuncture as Adjunctive Therapy for Chronic Stable Angina: A Randomized Clinical Trial. JAMA Intern Med 2019.

2. Benjamin EJ, Blaha MJ, Chiuve SE, Cushman M, Das SR, Deo R, De Ferranti SD, Floyd J, Fornage M, Gillespie C: Heart disease and stroke statistics-2017 update. 2017.

3. Khot AM, Anuradha HV, Prakash VS, Shivamurathy MC: Antianginal Efficacy and Tolerability of Ranolazine as an Add-on Drug to Concomitant Medications Primarily Metoprolol in Chronic Stable Angina Patients: A Prospective, Open-Label Study. Journal of pharmacology \& pharmacotherapeutics 2017, 8(1):21-27.

4. Vlachopoulos C, Georgakopoulos C, Pollalis D, Tousoulis D: Stable Angina Pectoris. Coronary Artery Disease: From Biology to Clinical Practice 2017:157.

5. Ohman EM: Chronic Stable Angina. New England Journal of Medicine 2016, 374(12):1167-1176.

6. Camero Y: Management of Coronary Artery Disease and Chronic Stable Angina. US Pharm 2017, 42(2):27-31.

7. Chong C-R, Ong GJ, Horowitz JD: Emerging drugs for the treatment of angina pectoris. Expert opinion on emerging drugs 2016, 21(4):365-376.

8. Jia Y, Leung S-w: Efficacy of Chinese herbal drugs for angina pectoris: frequentist network metaanalyses. The Lancet 2017, 390:S72.

9. Zhu X, Wu H-Y, Shaw P-C, Peng W, Su W: Specific DNA identification of Pheretima in the Naoxintong capsule. Chinese medicine 2019, 14(1):41.

10. Chan A-W, Tetzlaff JM, Altman DG, Laupacis A, Gøtzsche PC, Krleža-Jerić K, Hróbjartsson A, Mann H, Dickersin K, Berlin JA et al: SPIRIT 2013 statement: defining standard protocol items for clinical trials. 
Ann Intern Med 2013, 158(3):200-207.

11. Chan A-W, Tetzlaff JM, Gøtzsche PC, Altman DG, Mann H, Berlin JA, Dickersin K, Hróbjartsson A, Schulz KF, Parulekar WR et al: SPIRIT 2013 explanation and elaboration: guidance for protocols of clinical trials. BMJ 2013, 346:e7586.

12. [Guideline for diagnosis and treatment of patients with chronic stable angina (no abstract)]. Zhonghua xin xue guan bing za zhi 2007, 35(3):195-206.

13. Montalescot G, Sechtem U, Achenbach S, Andreotti F, Arden C, Budaj A, Bugiardini R, Crea F, Cuisset T, Di Mario C et al: 2013 ESC guidelines on the management of stable coronary artery disease: the Task Force on the management of stable coronary artery disease of the European Society of Cardiology. Eur Heart J 2013, 34(38):2949-3003.

14. Chinese Society of Cardiology CMA: Guideline for diagnosis and treatment of patients with chronic stable angina (no abstract). Zhonghua xin xue guan bing za zhi 2007, 35(3):195.

15. Fu M, Rosengren A, Thunström E, Mandalenakis Z, Welin L, Caidahl K, Pivodic A, Zhong Y, Ergatoudes C, Morales D et al: Although Coronary Mortality Has Decreased, Rates of Cardiovascular Disease Remain High: 21 Years of Follow-Up Comparing Cohorts of Men Born in 1913 With Men Born in 1943. Journal of the American Heart Association 2018, 7(9).

16. Roth GA, Johnson CO, Abate KH, Abd-Allah F, Ahmed M, Alam K, Alam T, Alvis-Guzman N, Ansari H, Ärnlöv J et al: The Burden of Cardiovascular Diseases Among US States, 1990-2016. JAMA cardiology 2018, 3(5):375-389.

17. Organization WH: Global status report on noncommunicable diseases 2014: World Health Organization; 2014.

18. Cao H, Zhai J, Li N, Cao H, Lei X, Mu W, Liu Z, Wang H, Shang H: CUPID: a protocol of a randomised controlled trial to identify characteristics of similar Chinese patent medicines. BMJ Open 2014, 4(11).

19. Kaski JC: Chronic Stable Angina Pectoris: History and Epidemiology. In: Essentials in Stable Angina Pectoris. edn.: Springer; 2016: 1-13.

20. Padala SK, Lavelle MP, Sidhu MS, Cabral KP, Morrone D, Boden WE, Toth PP: Antianginal Therapy for Stable Ischemic Heart Disease: A Contemporary Review. J Cardiovasc Pharmacol Ther 2017, 22(6):499-510.

21. Han J, Tan H, Duan Y, Chen Y, Zhu Y, Zhao B, Wang Y, Yang X: The cardioprotective properties and the involved mechanisms of NaoXinTong Capsule. Pharmacol Res 2019, 141:409-417.

22. Geng X, Chen L, Zhao B-C, Li C-X, Qiu L-Z, You X-Y, Wang Y-M, Zhang L-S, Wang H: [Preliminary investigation on mechanism of Naoxintong capsule's preventive treatment of cardio-cerebrovascular disease based on serum proteomics]. Zhongguo Zhong Yao Za Zhi 2016, 41(15):2864-2871.

23. Li W-X, Zhang S-Q, Zhao Y-D, Tang J-F, Li C-X, Wang X-Y, Li X-L: [Study progress on chemical compounds, pharmacological action and clinical application of Naoxintong capsule]. Zhongguo Zhong Yao Za Zhi 2018, 43(10):1998-2005.

24. Schulz KF, Altman DG, Moher D: CONSORT 2010 statement: updated guidelines for reporting parallel group randomised trials. BMJ 2010, 340:c332. 
Figures

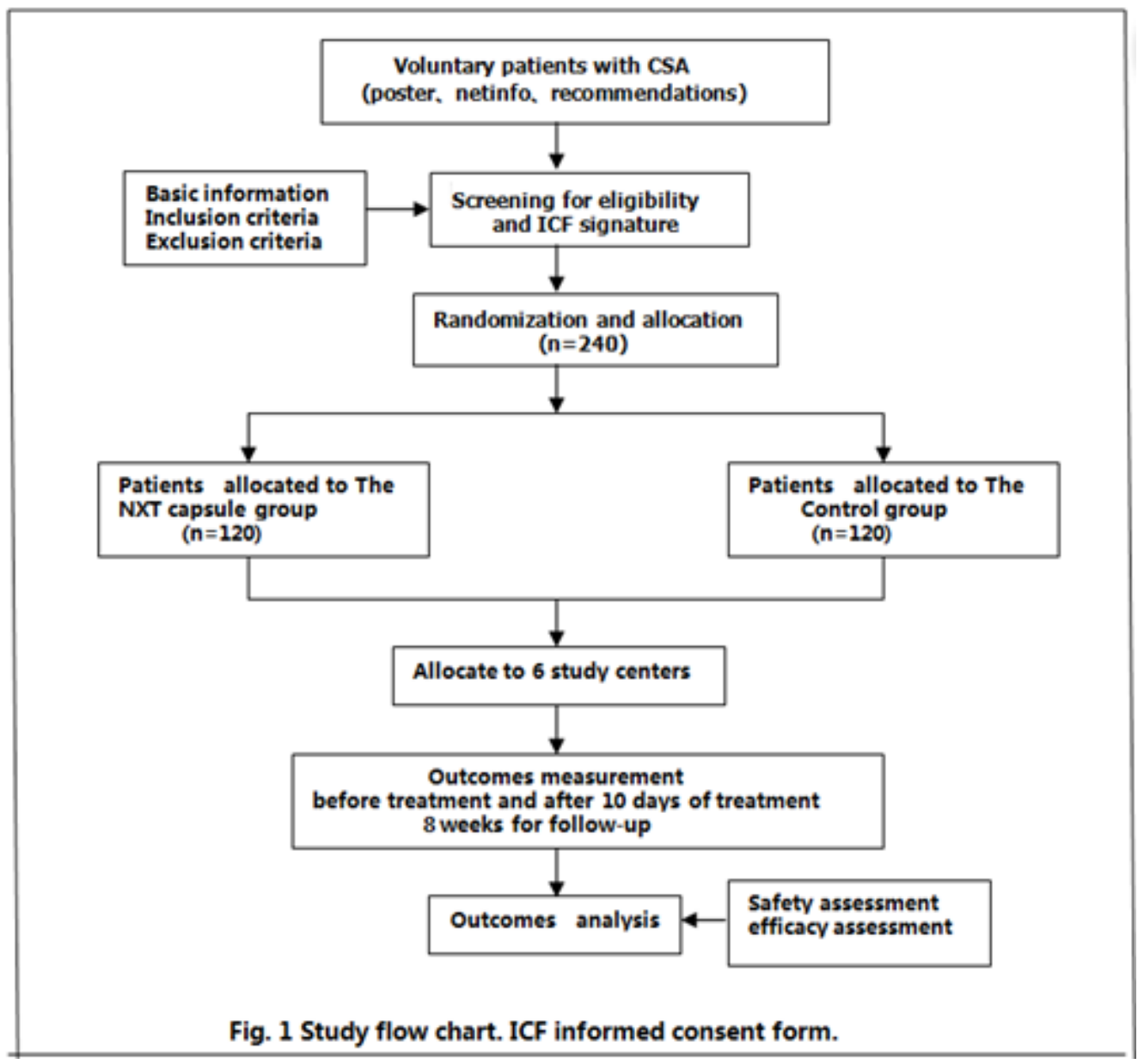

\section{Figure 1}

Study flow chart

\section{Supplementary Files}

This is a list of supplementary files associated with this preprint. Click to download.

- SPIRITFillablechecklist.doc 